\title{
Comparative in vitro activity of Meropenem, Imipenem and Piperacillin/tazobactam against 1071 clinical isolates using 2 different methods: a French multicentre study
}

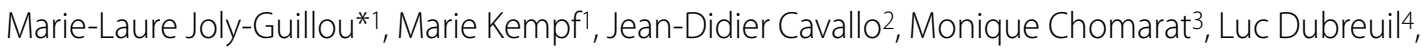 \\ Jeanne Maugein 5 , Claudette Muller-Serieys ${ }^{6}$ and Micheline Roussel-Delvallez ${ }^{7}$
}

\begin{abstract}
Background: Meropenem is a carbapenem that has an excellent activity against many gram-positive and gramnegative aerobic, facultative, and anaerobic bacteria. The major objective of the present study was to assess the in vitro activity of meropenem compared to imipenem and piperacillin/tazobactam, against 1071 non-repetitive isolates collected from patients with bacteremia (55\%), pneumonia (29\%), peritonitis (12\%) and wound infections (3\%), in 15 French hospitals in 2006. The secondary aim of the study was to compare the results of routinely testings and those obtained by a referent laboratory.

Method: Susceptibility testing and Minimum Inhibitory Concentrations (MICs) of meropenem, imipenem and piperacillin/tazobactam were determined locally by Etest method. Susceptibility to meropenem was confirmed at a central laboratory by disc diffusion method and MICs determined by agar dilution method for meropenem, imipenem and piperacillin/tazobactam.

Results: Cumulative susceptibility rates against Escherichia coli were, meropenem and imipenem: $100 \%$ and piperacillin/tazobactam: 90\%. Against other Enterobacteriaceae, the rates were meropenem: 99\%, imipenem: 98\% and piperacillin/tazobactam: 90\%. All Staphylococci, Streptococci and anaerobes were susceptible to the three antibiotics. Against non fermeters, meropenem was active on $84-94 \%$ of the strains, imipenem on $84-98 \%$ of the strains and piperacillin/tazobactam on $90-100 \%$ of the strains.
\end{abstract}

Conclusions: Compared to imipenem, meropenem displays lower MICs against Enterobacteriaceae, Escherichia coli and Pseudomonas aeruginosa. Except for non fermenters, MICs90 of carbapenems were $<4 \mathrm{mg} / \mathrm{L}$. Piperacillin/tazobactam was less active against Enterobacteriaceae and Acinetobacter but not $P$. aeruginosa. Some discrepancies were noted between MICs determined by Etest accross centres and MICs determined by agar dilution method at the central laboratory. Discrepancies were more common for imipenem testing and more frequently related to a few centres. Overall MICs determined by Etest were in general higher ( $0.5 \log$ to 1 log fold) than MICs by agar dilution.

\section{Background}

Antimicrobial susceptibility surveillance programmes represent one of the main recommendations to control resistant organisms, providing essential information in order to improve the quality of empiric antimicrobial prescribing or guiding development of antimicrobial poli-

\footnotetext{
* Correspondence: mljoly-guillou@chu-angers.fr

1 Bacteriology department, Universitary Hospital, (Larrey St), Angers (49000), France
}

() 2010 Joly-Guillou et al; licensee BioMed Central Ltd. This is an Open Access article distributed under the terms of the Creative Commons Attribution License (http://creativecommons.org/licenses/by/2.0), which permits unrestricted use, distribution, and reproduction in any medium, provided the original work is properly cited. cies. National and regional distributions of the data are important to enable local prescribing practices. The carbapenem (meropenem, imipenem)? with activity against P. aeruginosa have been the most active broad-spectrum antimicrobial class documented by numerous large surveillance programs [1]. One of the most cited global surveillance studies includes the Meropenem Yearly Susceptibility Test Information Collection (MYSTIC) program, an international resistance surveillance study 
which has been initiated 10 years ago with more than 100 participants worldwide [2-9]. The MYSTIC program is considered as a valuable tool to recognize the emergence of carbapenem and broad spectrum resistance mechanisms. An observatory surveillance system was established in 2006 to monitor the in vitro activity of meropenem following its approval in France in 2006 [10]. The major objective of the present study was to assess the in vitro activity of meropenem which has been recently re-introduced in French hospitals, compared to imipenem and piperacillin/tazobactam, against clinical isolates included in the spectrum of meropenem. One thousand and seventy one pathogens responsible for severe nosocomial infections were collected from 15 geographically diverse institutions in France. The secondary aim of the study was to compare the results of routinely testings and those obtained by a central laboratory.

The 15 French microbiology laboratories conducted susceptibility testing by disk diffusion test or broth dilution method according to the CA-SFM (Comite de l'Antibiogramme de la Société Française de Microbiologie) recommendations [11]. Since the participants of the 15 laboratories were using the CA-SFM breakpoints and because the EUCAST breakpoints will be introduced soon in France, approved CA-SFM and EUCAST (European Committee for Antimicrobial Susceptibility Testing [12]) interpretative breakpoints were used. The results obtained by the different laboratories (MICs by Etest method) were compared to those obtained by the centralized agar dilution method.

\section{Methods}

Fifteen French Community and Universitary Hospital Centres participated in this study and were chosen to represent geographically separate areas. Clinical isolates were collected between January and June 2006.

\section{Isolates}

The study protocol outlined specific quotas per medical centre among E. coli and other Enterobacteriaceae, non fermenter Gram-negative bacilli, Staphylococci, Streptococcus pneumoniae and anaerobes, for a total of 80 bacterial strains. Each centre collected the first 20 clinically relevant non-repetitive isolates of each selected species from patients hospitalized in 2006 with nosocomial infections: bacteremia, peritonitis, pulmonary infections and wound infections. Clinical and microbiological relevance was defined by "100 recommendations on nosocomial infections" from the French Health Ministry [13]. Species or genera known to be resistant to meropenem (methicillin-resistant -Staphylococci, Enterococccus faecium and Stenotrophomonas maltophilia) were excluded from the study collection.

\section{Antimicrobial susceptibility methods}

Antibiotic susceptibility testing was carried out using the local centre routine methods (Vitek, Etest, disk diffusion method). Susceptibilities were determined for meropenem, imipenem and piperacillin/tazobactam (8/1). Oxacillin susceptibility of Staphylococci was determined by each individual centre using routine methodology. The detail of each method was provided by each participant. Etest method was used by each participant in order to determine MICs for the following agents: meropenem, imipenem and piperacillin/tazobactam (tazobatam at one concentration of $4 \mathrm{mg} / \mathrm{L}$ ) according to the manufacturer's recommendations. All aerobic bacteria were tested onto Mueller Hinton agar. The medium was supplemented with $5 \%$ sheep blood for S. pneumoniae susceptibility testing. Susceptibilities of anaerobes were tested in a centralized laboratory. Meropenem, imipenem and piperacillin/tazobactam were tested by agar dilution method onto Brucella medium (DIFCO) according CLSI M11 A2 reference method and Etest method was realized on the carbapenems (meropenem, imipenem) only.

All bacteria were centralized and sent to a specific laboratory. Antimicrobial susceptibility testing was determined by Agar dilution reference method and disk diffusion tests using $30 \mu \mathrm{g}$ disks (Biorad Laboratories, France) were performed for meropenem according to the CA-SFM recommendations [11]. The antimicrobials were obtained from the manufacturers: meropenem (AstraZeneca Pharmaceuticals, France) imipenem (Merck Laboratory, France) piperacillin/tazobactam (Wyeth Pharmaceuticals, France). The antimicrobials were tested at the following dilution range: meropenem and imipenem: from 0.016 to $256 \mathrm{mg} / \mathrm{L}$; piperacillin/tazobactam: from $0.016 / 4$ to $1,024 / 4 \mathrm{mg} / \mathrm{L}$. MICs interpretative criteria followed published guidelines established by the CASFM (2006) or EUCAST (2006) where applicable. Quality controls including the following ATCC strains or CIP strains (Pasteur Institute Collection) were tested by each centre on the day of testing: E. coli ATCC 25922, S. pneumoniae CIP 104485, P. aeruginosa ATCC 27853, Staphylococcus aureus ATCC 25923.

\section{Beta lactamase screening}

When suspected, Gram negative bacilli were screened for Expanded-Spectrum-Beta-Lactamase activity (ESBL) according to the "100 recommendations on nosocomial infections" from the French Health Ministry [13]; EBSL activity was confirmed by in vitro synergy between third generation cephalosporins and clavulanate $(2 \mathrm{mg} / \mathrm{L})$. Hyper or depressed production of AmpC beta-lactamase was identified by cloxacillin and by high level resistance to cephalosporins and piperacillin/tazobactam, with no change in susceptibility in the presence of clavulanate. All P. aeruginosa and Acinetobacter with an elevated carbap- 
enem MIC result ( $\geq 2 \mathrm{mg} / \mathrm{L}$ ) were screened for the presence of a metallo $\beta$-lactamase or another serine $\beta$ lactamase, using Etest beta-lactamase strip (AB Biodisk laboratories: Etest + EDTA and Etest + clavulanate) in presence or in absence of a concentration of cloxacillin in order to inhibit the presence of AmpC cephalosporinase.

\section{Ethical conduct of the study}

The study has been conducted in accordance with "recommendations guilding physicians in biomedical research involving human patients " (declaration of Helsinki 1964). The study did not involved biological material or gentically modified organisms. All the strains involved in the study came from routine samples without any additionnal material.

\section{Statistical analysis}

The statistical significance tests of differences between centres and between the two methods were carried out using analysis of variance and Student's t test. MICs versus zone diameter scattergram was prepared for meropenem. Very major error (VME) was defined as a difference superior of $2 \log 2$ between the MICs carried out by the two methods, major error (ME) as a difference of $2 \log 2$ and minor error $(\mathrm{mE})$ as a difference of $1 \log 2$.

\section{Results}

A total of 1071 isolates were included in this study. They were isolated from bacteremia, (57\%) peritonitis (11\%), pneumonia $(13 \%)$ or bronchitis $(17 \%)$ and wound infections (2\%).

\section{In vitro susceptibility results}

Table 1 shows the ratio of susceptible strains to antibiotics, determined by the local study participants by routine methods: agar diffusion (56\%), broth dilution (VITEK [36\%] or ATB API [8\%]). Table 1 also compares the rates of susceptible strains, according to the CA-SFM criteria, obtained for meropenem, imipenem and piperacillin/ tazobactam, by the centralized agar dilution method and locally determined by Etest method.

Table 2 summarizes the MIC values inhibiting 50\% (MIC50), 90\% (MIC90) as well as the MIC range and the percentage of susceptible strains obtained by centralized agar dilution method according to EUCAST criteria. Figure 1 shows the concordance between disk zone diameter ( $\mathrm{mm})$ and MICs values for meropenem.

\section{Gram-negative pathogens}

Susceptibility testing, determined by local participants, showed that carbapenems were the most effective drugs against Enterobacteriaceae and Acinetobacter, whereas piperacillin/tazobactam was the most effective drug against other non fermenter bacilli. The incidence of ESBL producers was $2 \%$ among $E$ coli, $5 \%$ among other Enterobacteriaceae (2.5\% among Klebsiella pneumoniae) and $0 \%$ among all non fermenter bacilli. No metallo betalactamase has been identified in P. aeruginosa.

Results obtained by agar dilution method demonstrated the lowest MICs values with meropenem for Enterobacteriaceae. MICs of meropenem were frequently 2-fold lower than MICs of imipenem for Enterobacteriaceae and $P$. aeruginosa. MICs observed for carbapenems in nonfermenter Gram-negative bacilli were higher. Following EUCAST breakpoints, no E. coli resistant to carbapenems were identified. One Enterobacter cloacae isolate was resistant both to imipenem and meropenem $(\mathrm{MIC}=16$ $\mathrm{mg} / \mathrm{L}$ ) and two Proteus mirabilis showed an intermediate susceptibility to imipenem $(\mathrm{MICs}=4 \mathrm{mg} / \mathrm{L})$; all other Enterobacteriaceae were fully susceptible to carbapenems. Following EUCAST criteria, the same proportion of susceptible strains was observed for meropenem and imipenem. Among non fermenter bacilli other than $P$. aeruginosa, one Acinetobacter and one Alcaligenes were resistant to meropenem (MICs: $16 \mathrm{mg} / \mathrm{l}$ ) but respectively intermediate or susceptible (MIC respectively $8 \mathrm{mg} / \mathrm{l}$ and $2 \mathrm{mg} / \mathrm{l}$ ) to imipenem. Figure 1 displays the scattergram of meropenem MICs values versus disc diffusion zone diameters. Error rate (1.6\% of minor errors observed with Gram-negative bacilli) was within an acceptable range. Piperacillin/tazobactam demonstrated very good activity against Gram negative bacilli, particularly against non $P$. aeruginosa, aerobes, and bacilli. Discrepancies, which are the most important with that antibiotic, are related to the combination of piperacillin and tazobactam at various concentrations depending the method used.

\section{Gram-positive pathogens}

The in vitro activity of antimicrobials against Gram positive pathogens is displayed on Table 1. A total of 410 pathogens were tested. Table 2 gives the MIC50, the MIC90, the range and the percentage of susceptible strains. Against Gram-positive pathogens, imipenem frequently showed MICs one or two-fold lower than meropenem without any significant difference between the two compounds $(\mathrm{p}>0.05)$. All strains were susceptible to imipenem, meropenem and piperacillin/tazobactam.

\section{Anaerobes}

Figure 2 shows the MIC cumulative curves determined by agar dilution method. The best activity was observed with meropenem and imipenem $(\mathrm{MIC} 90=0.5 \mathrm{mg} / \mathrm{L})$, followed by imipenem $(\mathrm{MIC} 90=8 \mathrm{mg} / \mathrm{L})$.

\section{Method comparison for Carbapenem MICs results}

Comparison between agar dilution method and Etest method shows correlations of 0.72 for meropenem and 0.74 for imipenem. Table 3 illustrates that $40 \%(n=367)$ of the results were identical for meropenem and $23 \%$ (210) for imipenem. Respectively, for meropenem and imipenem, $81.4 \%$ and $61 \%$ of the strains tested were within +/- $1 \log 2(\mathrm{mE})$ dilution and $96.6 \%$ and $91.5 \%$ 
Trable 1: Susceptibility pattern of nosocomial infection agents obtained at local and central laboratories.

\begin{tabular}{|c|c|c|c|}
\hline \multirow[t]{3}{*}{ Drugs } & \multicolumn{3}{|c|}{ \% Susceptibility (Number tested) } \\
\hline & \multicolumn{2}{|c|}{ Locally determined } & \multirow{2}{*}{$\begin{array}{c}\text { Centralized } \\
\text { Agar DM }\end{array}$} \\
\hline & Routine & Etest & \\
\hline \multicolumn{4}{|l|}{ Escherichia coli } \\
\hline Meropenem & $100(92)$ & $100(139)$ & $100(139)$ \\
\hline Imipenem & $100(139)$ & $100(139)$ & $100(139)$ \\
\hline Piperacillin/tazobactam* & $82(139)$ & $89(139)$ & $90(139)$ \\
\hline \multicolumn{4}{|c|}{ Enterobacteriaceae (other than E coli) } \\
\hline Meropenem & $100(86)$ & $99(139)$ & $99(139)$ \\
\hline Imipenem & $99(139)$ & 97 (139) & $97(139)$ \\
\hline Piperacillin/tazobactam* & $78(139)$ & $86(139)$ & $99(139)$ \\
\hline \multicolumn{4}{|l|}{ Pseudomonas aeruginosa } \\
\hline Meropenem & $87(87)$ & $91(129)$ & $95(129)$ \\
\hline Imipenem & $78(137)$ & $70(129)$ & $84(129)$ \\
\hline Piperacillin/tazobactam* & $88(136)$ & $89(129)$ & $90(129)$ \\
\hline \multicolumn{4}{|l|}{ Acinetobacter } \\
\hline Meropenem & $88(50)$ & $87(86)$ & $95(79)$ \\
\hline Imipenem & $94(84)$ & $94(86)$ & $97(79)$ \\
\hline Piperacillin/tazobactam* & $79(82)$ & $80(86)$ & $100 *(79)$ \\
\hline \multicolumn{4}{|c|}{ Other non fermentative bacteria } \\
\hline Meropenem & $73(33)$ & $89(33)$ & $88(33)$ \\
\hline Imipenem & $85(41)$ & $87(33)$ & $88(33)$ \\
\hline Piperacillin/tazobactam* & $90(41)$ & $97(33)$ & $90(33)$ \\
\hline \multicolumn{4}{|c|}{ Staphylococcus aureus (susceptible to methicillin) } \\
\hline Meropenem & - & $100(145)$ & $100(145)$ \\
\hline Imipenem & - & $100(145)$ & $100(145)$ \\
\hline Piperacillin/tazobactam* & - & $100(145)$ & $100(145)$ \\
\hline \multicolumn{4}{|c|}{ Coagulase negative staphylococci (susceptible to methicillin) } \\
\hline Meropenem & - & $100(129)$ & $100(129)$ \\
\hline Imipenem & - & $100(129)$ & $100(129)$ \\
\hline Piperacillin/tazobactam* & - & $100(129)$ & $100(129)$ \\
\hline \multicolumn{4}{|l|}{ Streptococcus pneumoniae } \\
\hline Meropenem & - & $100(136)$ & $100(136)$ \\
\hline Imipenem & - & $100(136)$ & $100(136)$ \\
\hline Piperacillin/tazobactam* & - & $100(136)$ & $100(136)$ \\
\hline
\end{tabular}

\footnotetext{
*Tazobactam: 4 mg/L
} 


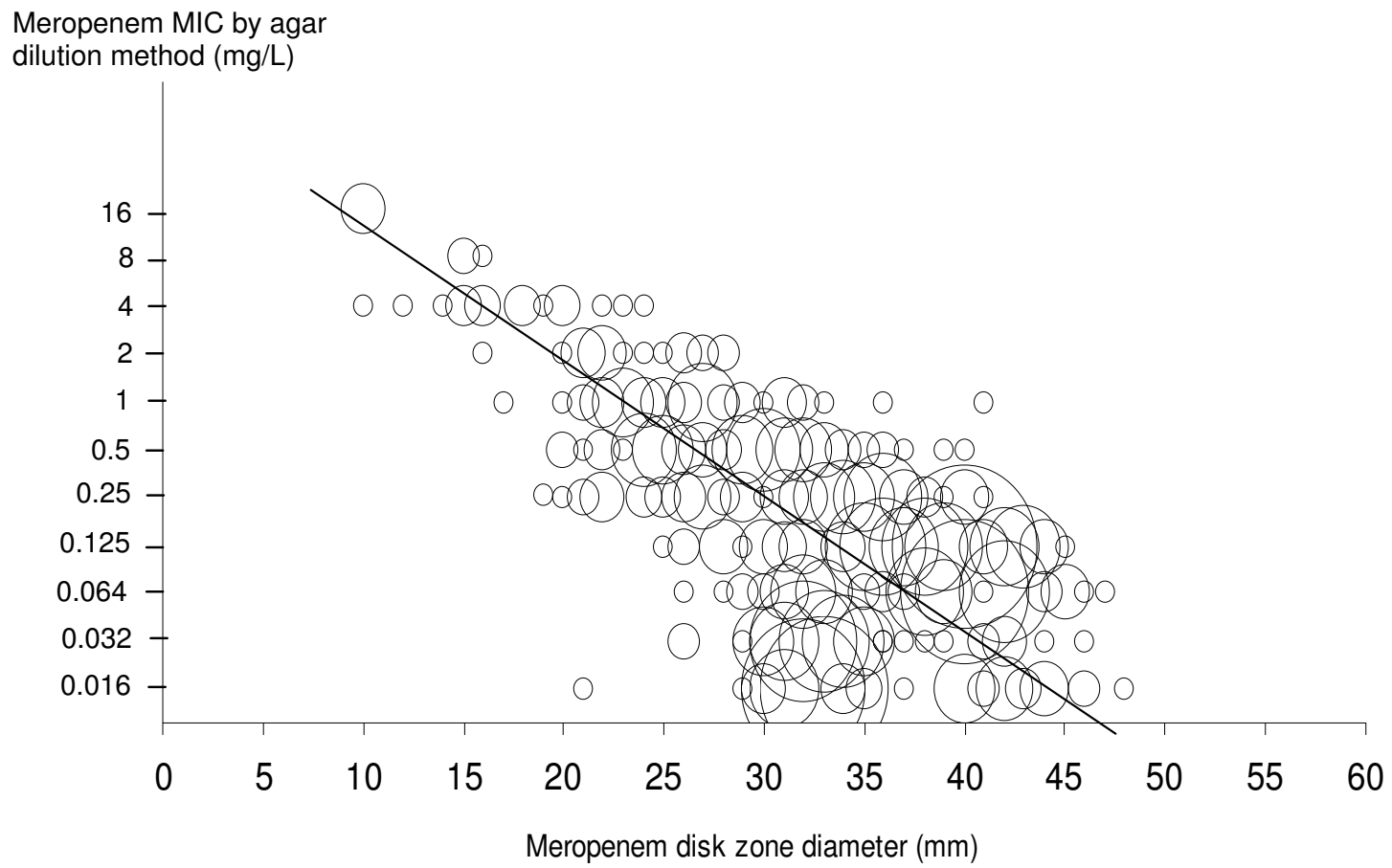

Figure 1 Scattergram comparing meropenem disk zone diameters and log MICs by using agar dilution method for 725 strains (EUCAST values). Minor errors figure in full circles.

ithin +/- 2log2 (ME) dilution. A significant trend toward a higher Etest MIC result was observed among these results for the two compounds $(\mathrm{p}<0.01)$. The trend toward a higher Etest MIC was higher for imipenem (1.1 $\log 2$ dilution) than for meropenem (0.5 log2 dilution).

\section{Factors influencing inter method categorical MIC errors}

Three percent of VME (31 strains) were observed for meropenem. Gram-negative bacilli were over represented with 27/31 strains causing VME. All bacterial groups were represented. We did not identify any influence of a centre effect. Among the 15 centres, 0 to 6 VME per centre were observed. The range of agreement among the 15 centres was $68 \%$ to $98 \%$ (within $+/-1 \log$ ).

Seventy nine ME (8.5\%) were observed with imipenem. Gram-negative bacilli (GNB) or Gram-positive cocci (GPC) were involved ( $8 \%$ of the GNB and $7 \%$ of the GPC). A significant centre effect was observed ( $p<0.01)$ : $57 \%$ of ME observed with GPC were due to 2 centres. Quality controls realized by each participant during the study allowed the identification of errors by the two local participants. Among the 15 centres, 10 had $0 \%$ to $5 \%$ of $\mathrm{ME}$, 3 had 7 to $16 \%$ of $\mathrm{ME}$ and had not been identified by the quality control. Two had more than $30 \%$ of ME.

\section{Discusion and Conclusions}

This study was the first national prospective surveillance study assessing antimicrobial activity of meropenem against recent clinical isolates from French hospitals. Antimicrobial susceptibility surveillance studies play a fundamental role in the fight to control resistant organisms. Harmonization between methods and interpretations are important to compare the results of various surveys located in different geographic areas $[14,15]$. The EUCAST group has provided progress toward harmonization of susceptibility testing methods used in European Nations and an international consensus on breakpoint definitions. Since 2008, the CA-SFM recommends the EUCAST methods and breakpoints. In this study, we report that meropenem, imipenem and piperacillin/ tazobactam are very active against Gram-negative bacilli, including Enterobacteriaceae, P. aeruginosa, Acinetobacter and other non fermenter-bacilli. The susceptibility data obtained from this multicentre study were similar to data previously published for studies conducted in Canada and other European countries $[9,16]$. Over the last 5 years, E coli susceptibility worldwide has shown a trend to decrease, in particular to fluoroquinolones and beta- 
Table 2: Antimicrobial activity of meropenem, imipenem and piperacillin/tazobactam against 1071 pathogens determined by agar dilution reference method.

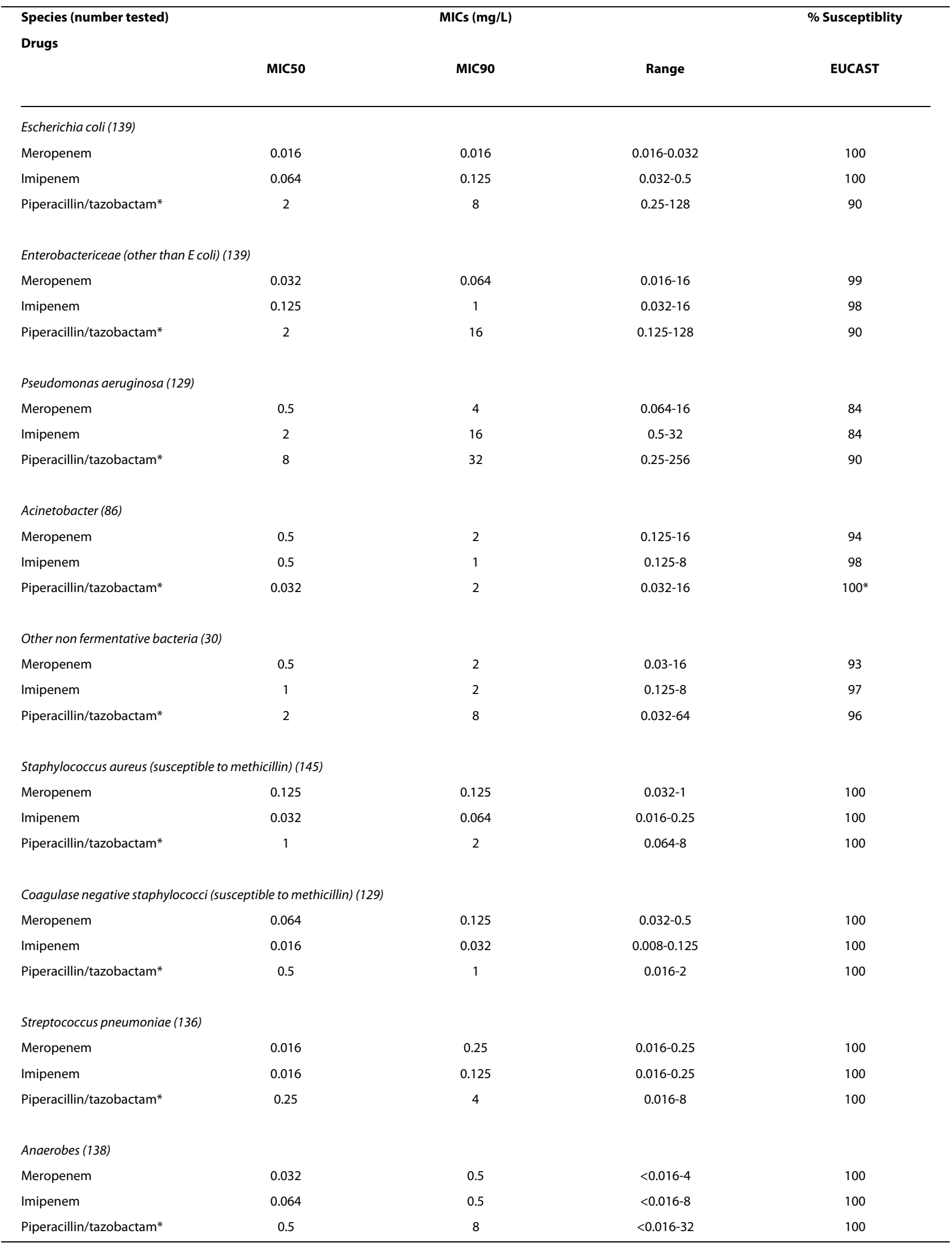

*Tazobactam: 4 mg/L 


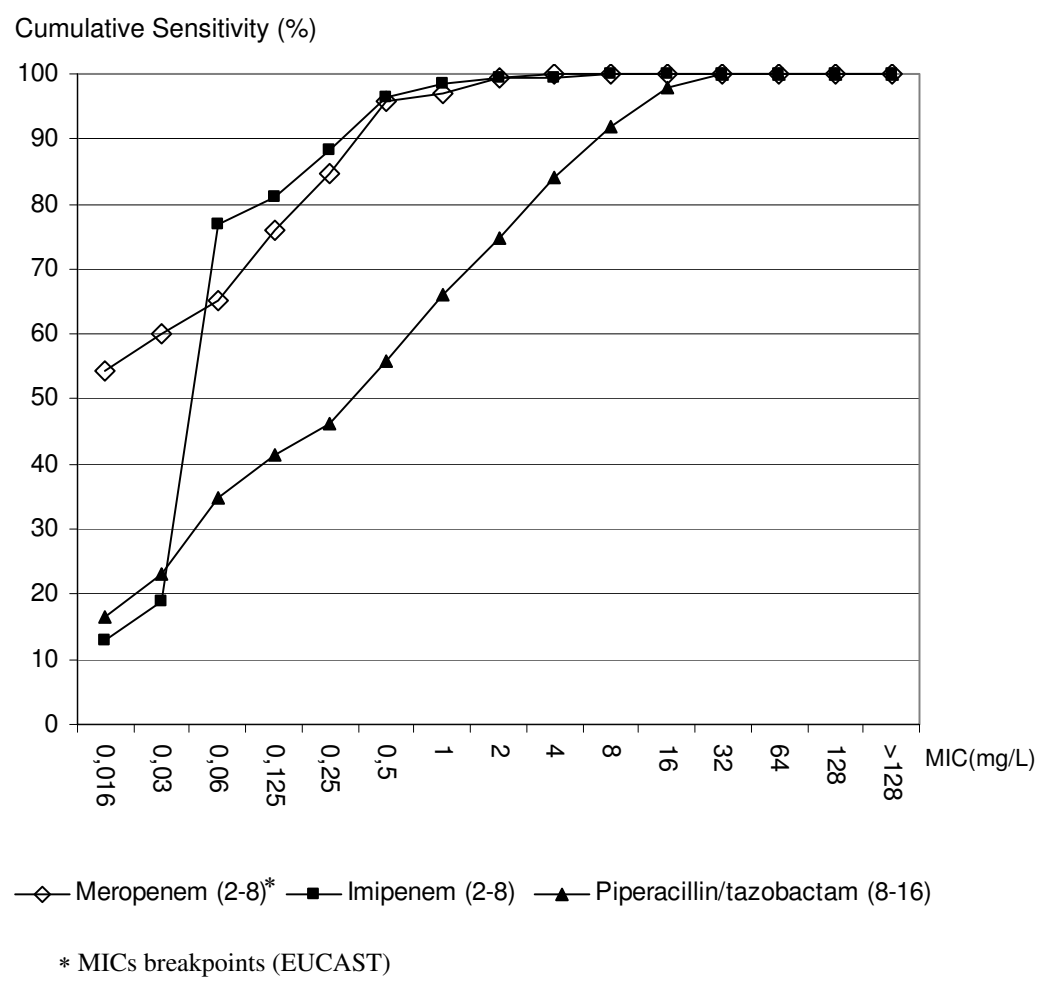

Figure 2 Comparative activity of antibiotics against anaerobes. Cumulative curves of MICs realized by agar dilution reference method.

actams, in relation with the dissemination of CTX-M producers and/or of an increase of AmpC [16-20]. Meropenem and imipenem demonstrate good activity against Enterobacteriaceae, including strains producing ESBLs or AmpC (100\% for E coli, 99\% for other Enterobacteriaceae), meropenem usually being 2 to 4 fold more potent than imipenem [21-23]. Susceptibility of Acinetobacter was close to 94 to $98 \%$ which is similar to other studies reporting susceptibility in Europe and the USA $[8,16]$. In this report, piperacillin/tazobactam was the most potent antibiotic against P.aeruginosa (90\% of susceptible strains versus $84 \%$ for carbapenems) as reported in other studies

Table 3: Variation of meropenem MIC and imipenem MIC results obtained by Etest method realized by local participants, and by agar dilution reference method realized by a centralized centre (anaerobes excluded).

$\%$ Variations from Agar Dilution Method MIC in log2 dilutions

\begin{tabular}{|c|c|c|c|c|c|c|c|c|}
\hline Etest & $\begin{array}{l}\text { VME } \\
>-2\end{array}$ & $\begin{array}{c}\text { ME } \\
-2\end{array}$ & $\begin{array}{c}\mathrm{mE} \\
-1\end{array}$ & Same & $\begin{array}{l}\mathrm{mE} \\
+1\end{array}$ & $\begin{array}{l}\text { ME } \\
+2\end{array}$ & $\begin{array}{l}\text { VME } \\
>+2\end{array}$ & Log trend * \\
\hline Meropenem $n=923$ & 7 & 9 & 78 & 367 & 306 & 132 & 24 & +0.5 \\
\hline (\%) & $(0.8)$ & $(2.2)$ & $(8.5)$ & $(40)$ & (33) & (14) & $(2.5)$ & \\
\hline Imipenem n $=923$ & 5 & 12 & 37 & 210 & 312 & 273 & 74 & +1.1 \\
\hline (\%) & $(0.5)$ & (1.3) & (4) & $(22.7)$ & (33.5) & $(29.7)$ & (8) & \\
\hline
\end{tabular}

* Trend in MIC when compared Etest/Agar dilution method

VME: very major error - ME: major error - $\mathrm{mE}$ : minor error 
[5]. Carbapenems as piperacillin/tazobactam showed a very good activity with low MICs against Gram-positive pathogens (MSSCN, MSSA and S. pneumoniae).

Communication of national and regional surveillance data are important to enable local prescribing practices, but in reality, every day antibiotic therapy is based on the sensitivity of antibiotic produced locally. Numerous studies have demonstrated the validity of the Etest method compared to the agar dilution method. The variability of inter-laboratory results is always tested by using reference strains but the statistical analysis of the centre results compared the global data is able to give rise to important informations, it reflects the reality of the daily work. The rate of agreement varies relatively to the antibiotic tested or the bacterial species [24-26]. The agreement (including minor errors) observed between the 2 testing methods was $>80 \%$ for meropenem, but was low for imipenem (61\%). The VME for meropenem were observed in Gram-negative bacilli, whereas there was no difference between Gram-negative bacilli and Gram-positive cocci for imipenem. In general, MIC values obtained using the Etest method were commonly higher than values obtained using agar dilution method. On occasion, interpretation of the MIC could have significant consequences on result reporting $(\mathrm{S}, \mathrm{I}, \mathrm{R})$ to the physician and subsequent prescribing. Analysis of the VME showed that no particular species was concerned but 2 centres presented VME $(\mathrm{p}<0.05)$. This observation suggested us that it could be due to a local technical problem such as inapropriate storage. Carbapenems and particularly imipenem are unstable antibiotics. The humidity could alter the quality of the strips and could modify the results.

\section{Competing interests}

The authors declare that they have no competing interests.

\section{Authors' contributions}

MLJG participated in the conception, study design, statistical analysis, coordination, supervision of laboratory analysis and manuscript writing. MK, JDC, MC, LD, JM, CMS and MRD participated in study design and supervision of laboratory testing. All authors read and approved the final manuscript

\section{Acknowledgements \\ Local participants of the study: G Arlet (AP HP Tenon, Paris), M Brun (Montpel- lier), H Chardon (Aix en Provence), F Jehl (Strasbourg). MC Ploy (Limoges), C Segond (Toulouse), M Vergnaud (Caen), V Vernet Garnier (Reims). This study was funded by an educational grant from Astra Zeneca (France).}

\footnotetext{
Author Details

'Bacteriology department, Universitary Hospital, (Larrey St), Angers (49000), France, 2Bacteriology department, Begin Military Hospital, (Paris Ave), St Mandé, (94163), France, ${ }^{3}$ Bacteriology department, Universitary Hospital LyonSud, Pierre Bénite, (69310), France, 4Faculty of Pharmacy, (Dr Laguesse St), Lille, (59006), France, ${ }^{5}$ Bacteriology department, Haut Leveque Hospital, (Magellan Ave), Pessac, (33604), France, ${ }^{6}$ Bacteriology department, Universitary Hospital Bichat Claude Bernard, AP-HP, Paris, (75018), France and 7Bacteriology department, Universitary Hospital Calmette, (J. Leclercq Bd), (59037), Lille, France
}

\section{References}

1. Zhanel GG, Wiebe R, Dilay L, Thomson K, Rubinstein E, Hoban DJ, Noreddin AM, Karlowsky JA: Comparative review of the carbapenems. Drugs 2007, 67:1027-1052.

2. Jones RN, Mendes C, Turner PJ, Masterton R: An overview of the meropenem yearly susceptibility test information collection (MYSTIC) Program: 1997-2004. Diagn Microbiol Infect Dis 2005, 53:247-256.

3. Unal S, Garcia-Rodriguez JA: Activity of meropenem and comparators against Pseudomonas aeruginosa and Acinetobacter spp. Isolated in the MYSTIC Program, 2002-2004. Diagn Microbiol Infect Dis 2005, 53:265-271

4. Masterton RG, Turner PJ: Trends in antimicrobial susceptibility in UK centres: the MYSTIC programme (1997-2002). Int J Antimicrob Agents 2006, 27:69-72.

5. Turner PJ: Meropenem and imipenem activity against Pseudomonas aeruginosa isolates from the MYSTIC Program. Diagn Microbiol Infect Dis 2006, 56:341-344.

6. Rhomberg PR, Deshpande LM, Kirby JT, Jones RN: Activity of meropenem as serine carbapenemases evolve in US Medical Centers: monitoring report from the MYSTIC Program. Diagn Microbiol Infect Dis 2006, 59:425-432

7. Rhomberg PR, Jones RN: Contemporary activity of meropenem and comparator broad-spectrum agents: MYSTIC program report from the Unites States component (2005). Diagn Microbiol Infect Dis 2007, 57:207-215.

8. Jones RN, Kirby JT, Rhomberg PR: Comparative activity of meropenem in US Medical Centers (2007): initiating the $2^{\text {nd }}$ decade MYSTIC Program surveillance. Diagn Microbiol Infect Dis 2008, 61:203-213.

9. Zhanel GG, DeCorby M, Nichol KA, Wierzbowski A, Baudry PJ, Karlowsky JA, Lagacé-Wiens P, Walkty A, Mulvey MR, Hoban DJ: Antimicrobial susceptibility of 3931 organisms isolated from intensive care units in Canada: Canadian National Intensive Care Unit Study, 2005/2006. Diagn Microbiol Infect Dis 2008, 62:67-80.

10. Joly-Guillou ML, Cavallo JD, Chomarat M, Dubreuil L, Maugein J, MullerSerieys C, Roussel-Delvallez M: Comparative activity of meropenem, imipenem and piperacillin/tazobactam against 1118 bacteria involved in hospital infections: a French multicentre study [Abstract]. 18th European Congress of Clinical Microbiology and Infectious Diseases Barcelona 2008:P612.

11. Members of the SFM Antibiogram Committee: Comité de I'Antibiogramme de la Société Française de Microbiologie, Report 2003. Int J Antimicrob Agents 2006, 21:364-391.

12. Kahlmeter G, Brown DF, Goldstein FW, MacGowan AP, Moutons JW, Odenholt I, Rodloff A, Soussy CJ, Steinbakk M, Soriano F, Stetsiouk O: European Committee on Antimicrobial Susceptibility Testing (EUCAST) Technical Notes on antimicrobial susceptibility testing. Clin Microbiol Infect 2006, 12:501-503.

13. Comité technique national des infections nosocomiales: 100 recommandations pour la surveillance et la prévention des infections nosocomiales. Ministère de l'Emploi et de la Solidarité Secrétariat d'Etat à la Santé et à l'action sociale; 1999.

14. Jones RN, Erwin ME, Hager HL, Hohl PE: Interlaboratory variations of fluoroquinolone susceptibility testing. An international study to validate the quality of microbiology results reported during the fleroxacin clinical trials. Diagn Microbiol Infect Dis 1993, 17:225-232.

15. Jones RN, Gordon KA, Biedenbach DJ: Comparisons of the in vitro susceptibility testing results for garenoxacin using six different national methods: report from the garenoxacin international bridging study. J Antimicrob Chemother 2004, 53:258-265.

16. Turner PJ: Meropenem activity against European isolates: report on the MYSTIC (Meropenem Yearly Susceptibility Test Information Collection) 2006 results. Diagn Microbiol Infect Dis 2008, 60:185-192.

17. Goossens H, Grabein B: Prevalence and antimicrobial susceptibility data for extended-spectrum $\beta$-lactamase- and Amp C-producing enterobacteriaceae from the MYSTIC Program in Europe and the United States (1997-2004). Diagn Microbiol Infect Dis 2005, 53:257-264.

18. Hawkey PM: The growing burden of antimicrobial resistance. J Antimicrob Chemother 2008, 62:1-9.

19. Patzer JA, Dzierzanowska D, Turner PJ: Trends in antimicrobial susceptibility of Gram negative isolates from a paediatric intensive care unit in Warsaw: results from the MYSTIC programme (1997-2007). J Antimicrob Chemother 2008, 62:369-375. 
20. Rossolini GM, D'Andrea MM, Mugnaioli C: The spread of CTX-M-type extended-spectrum beta-lactamases. Clin Microbiol Infect 2008, 14(Suppl 1):33-41.

21. Hernández JR, Velasco C, Romero L, Martiìnez-Martiinez L, Pascual A: Comparative in vitro activity of ertapenem against extended-spectrum beta-lactamase-producing Escherichia coli and Klebsiella pneumoniae isolated in Spain. Int J Antimicrob Agents 2006, 28:457-459.

22. Baldwin CM, Lyseng-Williamson KA, Keam SJ: Meropenem: a review of its use in the treatment of serious bacterial infections. Drugs 2008, 68:803-838

23. Kahlmete G: An international survey of the antimicrobial susceptibility of pathogens from uncomplicated urinary tract infections: the ECOSENS Project. J Antimicrob Chemother 2003, 51:69-76.

24. Nicodemo AC, Araujo MR, Ruiz AS, Gales AC: In vitro susceptibility of Stenotrophomonas maltophilia isolates: comparison of disc diffusion, Etest and agar dilution methods. J Antimicrob Chemother 2004, 53:604-608.

25. Muramatsu H, Ishikawa J, Nagura O, Horii T: Comparison of susceptibilities for fosfomycin determined by various methods in clinical isolates in 2003-2004. Jpn J Antibiot 2007, 60:107-124.

26. Vázquez JA, Arreaza L, Block C, Ehrhard I, Gray SJ, Heuberger S, Hoffmann S, Kriz P, Nicolas P, Olcen P, Skoczynska A, Spanjaard L, Stefanelli P, Taha MK, Tzanakaki G: Interlaboratory comparison of agar dilution and Etest methods for determining the MICs of antibiotics used in management of Neisseria meningitidis infections. Antimicrob Agents Chemother 2003, 47:3430-3434

\section{Pre-publication history}

The pre-publication history for this paper can be accessed here:

http://www.biomedcentral.com/1471-2334/10/72/prepub

doi: 10.1186/1471-2334-10-72

Cite this article as: Joly-Guillou et al., Comparative in vitro activity of Meropenem, Imipenem and Piperacillin/tazobactam against 1071 clinical isolates using 2 different methods: a French multicentre study BMC Infectious Diseases 2010, 10:72

Submit your next manuscript to BioMed Centra and take full advantage of:

- Convenient online submission

- Thorough peer review

- No space constraints or color figure charges

- Immediate publication on acceptance

- Inclusion in PubMed, CAS, Scopus and Google Scholar

- Research which is freely available for redistribution

Submit your manuscript at www.biomedcentral.com/submit
C Biomed Central 